\title{
Interprétation de l'agir et saisie des dimensions didactiques dans l'analyse des pratiques enseignantes
}

\author{
Ecaterina Bulea \\ Groupes GRAFE et LAF, Université de Genève \\ Ecaterina.Bulea@unige.ch
}

Cette contribution $^{1}$ vise à examiner les modalités sous lesquelles des dimensions didactiques de l'activité enseignante sont saisies dans le cadre d'analyses de pratiques outillées, c'est-à-dire dans le cadre de dispositifs délibérément mis en place pour susciter un retour réflexif des enseignants sur leur activité. La première partie de l'article rappelle brièvement la visée générale, les orientations et les dispositifs associés à l'analyse des pratiques. La deuxième partie précise le contexte de la recherche sur laquelle nous nous appuyons, le type de données examinées et le cadre méthodologique de recueil de ces données. La troisième partie présente la méthodologie d'analyse des données, résolument ancrée dans une perspective textuelle/discursive, qui réexploite le modèle de l'architecture textuelle élaboré dans le cadre de l'interactionnisme socio-discursif (voir Bronckart, 1997) en l'adaptant à l'examen des textes issus d'analyses de pratiques. La quatrième partie est centrée sur les caractéristiques du processus d'interprétation par les enseignants de leur activité, en mettant en évidence les configurations interprétatives (ou les « figures d'action ») qu'ils mobilisent dans leur discours. Enfin, la cinquième partie examine la place que ces configurations interprétatives laissent aux aspects didactiques, notamment : aux savoirs à enseigner ou enseignés, aux gestes didactiques, aux moyens d'enseignement.

\section{L'analyse des pratiques : visée, orientations, dispositifs}

L'analyse de l'activité (ou du travail, ou des pratiques - nous reviendrons plus loin sur cette indécision terminologique) a pour caractéristique principale d'être une démarche de réflexion, effectuée par les professionnels au sujet de leurs propres activités de travail et/ou de leurs pratiques. Prenant plusieurs formes et se réalisant en divers dispositifs méthodologiques, sa visée majeure concerne la compréhension de ces activités par les professionnels au travers d'un processus de prise de conscience de (certaines des) propriétés de celles-ci; et, sur cette base, le développement des capacités d'agir des personnes concernées, ainsi que la transformation, en principe positive, des organisations socioprofessionnelles dans lesquelles ces personnes sont impliquées. Que ce soit dans le champ de l'intervention en milieu de travail ou dans celui de la formation, cette démarche suscite actuellement un intérêt grandissant. Ses diverses formes de mise en œuvre s'accompagnent en outre de questionnements et de développements théoriques, méthodologiques et techniques variés, mais elles sont toutes sous-tendues par le postulat de l'efficacité développementale et du pouvoir transformateur et formateur de l'acte même d'interprétation de l'agir.

Notons encore que, comme le souligne Jobert, lorsqu'elle est intégrée dans des dispositifs de formation, l'analyse des pratiques contribue notablement à la mise en œuvre d'une forme de rapport, ou d'articulation, entre deux domaines traditionnellement non reliés, à savoir le domaine du travail et celui de la formation; cette mise en rapport exploitant fondamentalement (mais non exclusivement) le discours, ou l'activité langagière :

\footnotetext{
Les pratiques formatives regroupées sous l'appellation "d'analyse des pratiques" tentent de jeter des ponts entre l'espace et le temps de l'action productive, et ceux de la réflexion de et sur l'action. Ces modalités, largement utilisées en perfectionnement des formateurs ou d'enseignants, s'efforcent d'actualiser par le discours, dans l'ici et maintenant de la situation de formation, l'acte professionnel posé avant et ailleurs. (Jobert, 2002, p. 254, les italiques sont de nous)
} 
Comme en atteste la pluralité des expressions utilisées dès la première ligne de cette partie de l'article, et en dépit de sa visée formative et/ou développementale générale, l'analyse des pratiques ne désigne pas une approche homogène, ni du point de vue de ses ancrages théoriques, ni du point de vue des modalités de conceptualisation du travail qui lui sont sous-jacentes, ni du point de vue de la conception du langage explicitement ou implicitement mobilisée. Diverses variantes peuvent en être identifiées aujourd'hui, parmi lesquelles on peut citer le "praticien réflexif» (Schön, 1983/1994), la «clinique de l'activité » (Clot \& Faïta, 2000 ; Clot, 2001), la «psychodynamique du travail» (Dejours, 1999), les démarches «d'explicitation » (Vermersch, 1994), les mises en «récit autobiographique de l'expérience » (Pineau, 1985 ; Dominicé, 1990), etc. Ces variantes intègrent ou s'inspirent de disciplines différentes, notamment l'ergonomie (Daniellou, 1996), la psychologie (Clot, op. cit.; Dejours, op. cit.), la sociologie, ou encore la sociolinguistique (voir les travaux du réseau «Langage \& travail»: Boutet, 1995; Grosjean \& Lacoste, 1999 ; Borzeix \& Fraenkel, 2001); enfin, elles sont contrastées du point de vue des ancrages épistémologiques (phénoménologie, psychologie constructiviste d'inspiration piagétienne, psychologie développementale d'inspiration vygotskienne, etc.) et ont progressivement donné naissance à un ensemble de méthodes et techniques d'analyse de l'activité, parmi lesquelles : l'entretien d'explicitation (Vermersch, op. cit), les auto-confrontations simples et croisées (Clot \& Faïta, op. cit. ; Clot, op. cit.), l'instruction au sosie (Clot \& Soubiran, 1999 ; Scheller, 2000), etc. Notons encore que depuis les années 1980, et parallèlement à la création de dispositifs spécialement conçus pour l'analyse des pratiques comme ceux mentionnés ci-dessus, on constate l'exploitation des entretiens de recherche " classiques » ou semi-dirigés (Blanchet et al., 1985 ; Blanchet \& Gotman, 1992) à des fins explicites d'analyse des pratiques, ces entretiens accompagnant ou non des démarches d'observation du travail réel (voir Bronckart \& Groupe LAF, 2004 ; Bulea 2007, 2010).

S'agissant spécifiquement du travail enseignant, on peut constater a posteriori que l'analyse des pratiques ne s'est pas déployée dans une perspective didactique au sens strict du terme. Bien que l'activité réelle des enseignants ait toujours été au centre, les « verbalisations » suscitées chez ces derniers ne visaient pas spécialement un retour réflexif sur leur travail, mais plutôt la mise en évidence de l'expertise professionnelle de l'enseignant (Goigoux, 2002), ou encore de la dimension de «travail» de l'activité enseignante, conjointement à la contestation de ce que l'enseignement serait une "mission » ou un « don ». Dès lors, l'analyse des pratiques enseignantes, ou, plus exactement, l'analyse de l'activité des enseignants (Bernié \& Goigoux, 2005), a de fait surtout concerné la compréhension et la modélisation de gestes professionnels (Sensevy, 2005; Jaubert \& Rebière, 2006; Bucheton, 2009), l'analyse des répertoires et des postures qui font la spécificité du travail enseignant en tant qu'interaction (Cicurel, 2011), ou encore l'analyse des dimensions ergonomiques de l'activité enseignante et du rapport de celleci à la prescription (Saujat, 2002).

L'analyse et la conceptualisation des dimensions didactiques ont été, pour leur part, prises en charge par des recherches mobilisant des données recueillies in situ, en classe, la plupart du temps sous forme d'enregistrements vidéo (voir les recherches relatées dans Schneuwly \& Dolz, 2009 ; Canelas-Trevisi, 2009 ; Aeby Daghé \& Dolz, 2008 ; Sensevy, 1998, entre autres), mais qui ne comportent pas pour autant de volet d' «analyse des pratiques » au sens propre du terme, car elles ne mobilisent pas de dispositifs sollicitant explicitement la réflexivité des enseignants, comme ceux évoqués plus haut ; autrement dit, dans ce cadre, si les données enregistrées en classe servent bien entendu, et de manière incontestablement éclairante, à l'analyse didactique, cette dernière est le produit du travail des chercheurs et non celui de l'interprétation de l'activité par des praticiens.

Sans nullement hiérarchiser ou remettre en question la pertinence intrinsèque de chacune de ces perspectives de recherche rapidement esquissées ci-dessus, c'est dans la direction d'une mise en interaction de celles-ci que notre contribution se propose, en toute modestie, vu l'ampleur et la complexité de la tâche, de faire un pas: ainsi, nous examinerons dans ce qui suit, d'une part, les modalités d'interprétation de l'activité par les enseignants, attestables dans le cadre de dispositifs d'analyses de pratiques outillés; d'autre part, les conditions d'émergence, dans ce cadre interprétatif, de dimensions didactiques ${ }^{2}$ de l'activité enseignante. 


\section{Indications méthodologiques concernant le dispositif de recueil de données}

La recherche sur laquelle nous nous appuyons concerne des analyses de pratiques conduites avec des professeurs de français langue étrangère (FLE). Les données ont été recueillies dans trois institutions d'enseignement du FLE, qui se différencient en fonction de leur situation géographique, de leur statut, du type de public apprenant qui les fréquente et de la finalité de l'appropriation/acquisition de la langue par les apprenants. La première institution (désormais I1) accueille des apprenants adultes originaires de divers pays, vivant en Suisse, qui se trouvent souvent en situation de précarité économique, sociale et/ou politique (chômage, statut de réfugié, etc.) et dont le niveau de formation et d'acculturation à l'écrit et au travail intellectuel est faible ou très faible. La deuxième institution (désormais I2) se caractérise par un public apprenant constitué de fonctionnaires européens, en situation d'emploi confortable, voire privilégiée, ayant un niveau de formation élevé (universitaire), habitués de ce fait au travail intellectuel, et présentant des préoccupations relatives au monde du travail en contexte européen. La troisième institution (désormais I3) accueille un public constitué d'étudiants d'universités du monde entier, plutôt jeunes, d'un niveau d'éducation supérieure, maîtrisant ainsi les instruments du travail intellectuel, et qui souhaitent poursuivre des études universitaires en francophonie, dans différentes branches.

Neuf formateurs (huit femmes et un homme) ont accepté de participer à cette recherche. Ces personnes ont été sollicitées en raison de leur appartenance institutionnelle (trois formateurs par institution), mais aussi de leur disponibilité et de leur intérêt pour la démarche mise en place ${ }^{3}$. Il ne s'agit donc pas d'un échantillon représentatif, mais d'un groupe illustratif de la profession enseignante de FLE.

La démarche utilisée comporte une innovation méthodologique, en ce qu'elle relève d'un dispositif hybride, combinant « entretien de recherche » et « instruction au sosie ».

Méthode bien connue et très répandue en sciences sociales, l'entretien de recherche a été élaboré vers la fin de années 1920 dans le domaine de la psychologie sociale. Il a été mis en œuvre dans les enquêtes conduites par Roethlisberger et Dickson à la Western Electric (voir Blanchet et al., 1985), portant initialement sur les conditions matérielles de la productivité en entreprise, ensuite sur l'influence du «moral» des ouvriers sur cette même productivité. Actuellement, les définitions et les usages de l'entretien de recherche sont multiples; néanmoins, la plupart des auteurs soulignent: - la visée heuristique de l'entretien ; - son inscription dans un contexte de recherche; - enfin son aspect «objectivant» eu égard aux faits psychologiques et sociaux, dont il s'agit d'élaborer un savoir transmissible (Blanchet \& Gotman, 1992).

L'instruction au sosie est une méthode mise en place dans les années 1970, aux usines FIAT de Turin (voir Oddone, Re \& Briante, 1981). Elle a été reprise et transformée dans le cadre de la clinique de l'activité (Clot \& Soubiran, 1999; Scheller, 2000), dans une perspective développementale d'orientation vygotskienne. Cette méthode comporte trois étapes : - une étape d'entretien d'instruction d'abord, qui se déroule selon une consigne précise ["Suppose que demain je sois ton sosie et que je me trouve en situation de devoir te remplacer dans ton travail. Quelles sont les consignes que tu devrais me transmettre afin que personne ne remarque la substitution? 》], et lors de laquelle le travailleur (ou « modèle ») décrit le plus finement possible les propriétés effectives ou possibles de son activité de travail et les transmet ainsi à un remplaçant fictif (ou « sosie »); - une étape de transcription de cet entretien par le «modèle»; - une étape de production d'un commentaire écrit de la part du même " modèle » ayant trait à ses propres dires pendant l'entretien d'instruction. On le constate, l'objet visé par cette technique est l'activité de travail, à la fois sous l'angle de l'expérience (les « ficelles du métier ») et dans ses menus détails ; et la reconstruction de significations à propos de l'activité a lieu sous une modalité de projection d'abord (l'entretien d'instruction), et une modalité d'auto-confrontation ensuite (transcription et commentaire de l'entretien d'instruction).

Le dispositif que nous avons mis en place comporte deux parties se déroulant en continuité : la première, prenant la forme d'entretien semi-directif, et abordant, selon un canevas préétabli, des thèmes liés au parcours professionnel du formateur, à son expérience d'enseignement et aux caractéristiques du public 
auquel il s'adresse; la seconde, prenant la forme d'instruction au sosie (plus exactement de la partie « entretien d'instruction » de celle-ci) et abordant un agir particulier, délimité : l'enseignement du passé composé (nous reviendrons au point 5 sur les raisons du choix de cet objet d'enseignement). En respectant la technique propre à l'instruction au sosie, cette partie a demandé, de la part de l'interviewé, et par rapport à la partie précédente, un délicat exercice de décentration : le propre de l'instruction au sosie est de mettre en scène un agir hypothétique (celui du sosie remplaçant), mais dont la construction se fonde sur l'expérience passée de l'interviewé-modèle et sur son contexte professionnel réel (voir Bulea, 2010, pour une analyse). Au plan technique encore, l'intervieweur-sosie pose ses questions en «je » [Donc je termine la leçon / je leur dis quoi pour terminer ${ }^{4}$ ?], alors que l'interviewé-modèle est invité, et censé toujours ${ }^{5}$, répondre en utilisant un pronom de deuxième personne, «tu » ou « vous » [Alors / déjà tu vas leur donner / un / du travail (...) dans le livre d'exercices 3 page 45 euh ensuite euh tu peux aussi également les faire écrire un petit texte... - entretien avec VH].

Ces entretiens ont été conduits dans une perspective explicite d'analyse de l'activité et l'hybridation méthodologique à laquelle nous avons procédé était motivée notamment par le souhait de se donner les moyens de comparer et de contraster la manière dont un seul et même enseignant saisit des éléments de son activité selon des formats discursifs et interactif différents, afin d'examiner la possible influence de ces formats sur l'interprétation de l'agir produite.

Relevons encore que la recherche dont ces données sont issues comporte plusieurs volets : outre celui concerné par la présente contribution (voir aussi Bulea \& Jusseaume, sous presses), on a envisagé un volet centré sur l'influence du type de public apprenant sur les représentations que les formateurs se font de leur métier (voir Jusseaume, 2012), un autre centré sur l'influence du type de dispositif sur les modalités de saisie de l'agir, enfin un autre encore, abordant de manière comparative les aspects pédagogiques et didactiques du métier selon les caractéristiques de chacune des institutions (les analyses sont en cours). Nous ne pourrons pas rendre compte ici de tous ces aspects; en revanche, nous contrasterons ponctuellement nos analyses avec des résultats issus d'une recherche précédente, portant sur l'interprétation du travail infirmier (voir Bulea, 2007 et 2010), afin, d'une part, de mieux cerner certaines des spécificités du travail enseignant et de son interprétation, d'autre part de rendre compte, pardelà les différences entre métiers, des éventuels éléments de continuité ou de similitude liés au processus même d'interprétation de réalités à caractère praxéologique.

\section{Méthodologie d'analyse des données}

Pour l'analyse des données, la méthodologie utilisée exploite le modèle de l'architecture textuelle élaboré dans le cadre de l'interactionnisme socio-discursif (voir Bronckart, 1997 ; 2008) et relève d'une approche résolument textuelle-discursive. D'une part, nous considérons comme point de départ le texte, en l'occurrence l'entretien dans sa totalité et sa dynamique, en tant qu'unité communicative englobante et articulée à la forme d'activité humaine que constitue la recherche; texte dont il s'agit d'examiner les propriétés selon une perspective doublement «descendante » : d'une part en allant depuis les conditions de production du texte vers le texte comme produit, d'autre part en allant de l'unité texte vers les paliers inférieurs de structuration: segments thématiques et types de discours qui les organisent $\rightarrow$ relations prédicatives, mécanismes de connexion et de cohésion, mécanismes de prise en charge énonciative $\rightarrow$ unités minimales de l'ordre du mot.

L'élaboration d'une démarche d'analyse de textes commentant l'agir (divers documents institutionnels et entretiens avec les travailleurs notamment, voir Bronckart \& Groupe LAF, 2004 ; Bronckart \& Machado, 2005) a démarré lors de la recherche évoquée ci-dessus, portant sur le travail infirmier (voir à ce sujet Bulea \& Fristalon, 2004 ; Bronckart, Bulea \& Fristalon, 2004), et elle s'est prolongée et affinée par la suite lors de recherches portant sur le travail enseignant. Pour ce qui concerne les textes d'entretiens, cette démarche d'analyse présente les étapes et les caractéristiques qui suivent. 


\subsection{L'examen de la dynamique générale de l'entretien}

Conformément à la perspective "descendante » adoptée, la première étape concerne l'examen de la dynamique générale de l'entretien et a pour objectif de fournir une description conceptualisée de cet entretien tel qu'il s'est construit temporellement et interactivement. Cette étape comporte plusieurs opérations. D'abord, en nous centrant sur la distribution des tours de parole, nous cherchons à identifier les modes d'articulation de deux grandes catégories de segments :

- des segments d'introduction, de présentation ou d'amorce d'un thème, qui ont été qualifiés de segments d'orientation thématique;

- des segments produits par l'interviewé, généralement suite à une intervention de l'intervieweur, dans lesquelles un thème est effectivement traité, et qui sont qualifiées de segments de traitement thématique.

S'agissant des segments d'orientation thématique, d'un point de vue interactif, certains sont monogérés, c'est-à-dire sont exclusivement constitués d'interventions de l'intervieweur; d'autres sont polygérés c'est-à-dire comportent des interventions croisées de l'intervieweur et de l'interviewé, à caractère de négociation et/ou de recherche d'intercompréhension. Quant aux segments de traitement thématique, ils peuvent prendre des formes diverses : reformulation, extension et complexification, ou focalisation sur un aspect considéré comme pertinent, exemplification, etc.

La deuxième opération consiste à tenter d'identifier l'ensemble des thèmes traités au cours de l'entretien, et d'en proposer un étiquetage et une organisation « lisible».

Basée sur les deux opérations précédentes, la troisième consiste alors en la constitution du scénario de chaque entretien, qui résume et visualise, de manière globale, l'enchaînement temporel des thèmes tel qu'il résulte des interactions entre «orientation» et «traitement»; scénario qui restitue ainsi les conditions contextuelles chrono-logiques de l'élaboration langagière des représentations de l'agir concerné, et qui constitue une première base possible pour des comparaisons entre entretiens.

\subsection{L'examen des propriétés linguistiques des segments de traitement thématique}

La seconde étape de l'analyse concerne tout particulièrement les segments de traitement thématique, produits par l'interviewé, qui portent de manière explicite sur la tâche ou le travail concerné par l'entretien. A ces segments, sont appliquées un second ensemble d'opérations, inspirées du modèle de l'architecture textuelle proposé au sein de l'interactionnisme socio-discursif.

\subsubsection{L'identification des types de discours}

La première opération consiste en l'identification des types de discours qui (et tels qu'ils) se manifestent concrètement dans les entretiens. Les types de discours sont des modalités de gestion énonciative du contenu thématique, qui réalisent dans une langue donnée, et donc selon les ressources de cette même langue, des «attitudes énonciatives » tendanciellement universelles, qualifiées de mondes discursifs. La construction de ces derniers repose sur des opérations à caractère décisionnel, qui peuvent être résumées comme suit (pour une description détaillée, voir Bronckart, 1997): soit les coordonnées du monde sémiotisé dans le texte sont explicitement mises à l'écart de celles de la situation de production de celuici, opération qui correspond à l'ordre du «raconter » et qui se réalise par la création d'une origine temporelle (il y a quatre ans, hier, en 1857, etc.), soit cette disjonction n'est pas opérée, ce qui correspond à l'ordre de l'« exposer ». Corrélativement, soit les instances d'agentivité sémiotisées dans le texte sont mises en rapport avec la situation de production de celui-ci, ce qui correspond à un rapport d'«implication », marqué notamment par des unités pronominales déictiques, soit elles ne le sont pas, ce qui correspond à un rapport d' « autonomie ». Les mondes discursifs procèdent du croisement du résultat de ces décisions, qui produit quatre formats de base : «raconter impliqué », « raconter autonome», « exposer impliqué », et « exposer autonome ». Et ces mondes ainsi définis sont réalisés linguistiquement 
par les types de discours évoqués, respectivement le récit interactif, la narration, le discours interactif et le discours théorique. Dans les textes empiriques, ces types de discours peuvent évidemment se présenter de manière plus ou moins «pure », pouvant se superposer, fusionner, s'interpénétrer selon des modalités diverses et complexes. De même, il est important de souligner que le marquage des mondes discursifs (et donc la teneur effective des types de discours) varie d'une langue à l'autre. Pour la langue française, les principales propriétés spécifiques de chacun de ces types sont les suivantes.

a. Discours interactif

- Présence de phrases non déclaratives (interrogatives, impératives, exclamatives) ;

- Présence d'unités déictiques renvoyant à certains objets accessibles aux interactants ou à l'espace-temps de l'interaction (ostensifs, déictiques spatiaux et temporels);

- Présence de noms propres, ainsi que de pronoms et déterminants possessifs de 1ère et 2ème personne du singulier et du pluriel ;

- Présence de l'auxiliaire de mode pouvoir, ainsi que d'autres auxiliaires à valeur pragmatique, du type vouloir, devoir, falloir, etc.

b. Discours théorique

- Absence de phrases non déclaratives ;

- Absence d'unités déictiques renvoyant à certains objets accessibles aux interactants ou à l'espace-temps de l'interaction ;

- Absence de noms propres, ainsi que de pronoms et déterminats de 1ère et 2ème personne du singulier à valeur clairement exophorique ;

- Présence de multiples organisateurs à valeur logico-argumentative (mais, or, d'une part, d'autre part, en effet, etc.) ;

- Présence de nombreuses modalisations logiques, ainsi que l'omniprésence de l'auxiliaire de mode pouvoir.

c. Récit interactif

- Absence de phrases non déclaratives ;

- Présence d'organisateurs temporels (adverbes, syntagmes prépositionnels, coordonnants, subordonnants, etc.) ;

- Présence de pronoms et déterminants possessifs de 1ère et 2ème personne du singulier et $d u$ pluriel, qui renvoient directement aux protagonistes de l'interaction verbale ;

- Présence dominante d'anaphores pronominales, parfois associées à des anaphores nominales.

d. Narration

- Absence de phrases non déclaratives ;

- Présence d'organisateurs temporels (adverbes, syntagmes prépositionnels, coordonnants, subordonnants, etc.) ;

- Absence de pronoms et déterminants possessifs de 1ère et 2ème personne du singulier et $d u$ pluriel, qui renvoient directement aux protagonistes de l'interaction verbale ;

- Présence conjointe d'anaphores pronominales et d'anaphores nominales, ces dernières se présentant généralement sous la forme d'une reprise du syntagme antécédent, avec substitution lexicale.

Dans les textes du genre entretien, le type de discours «narration » est quasi toujours absent. On relève par contre des occurrences de récit interactif et de discours théorique, mais le type le plus fréquent est, bien évidemment, le discours interactif, qui peut prendre soit la forme d'un adressage concret entre les interlocuteurs, soit l'une des formes possibles de discours rapporté. On constate en outre la présence de formes discursives mixtes, ou hybrides, la plus fréquente étant celle du mixte théorique-interactif.

Notons encore que la distinction entre "genre textuel» et «type de discours" nous paraît ici fondamentale, en ce qu'elle permet de voir et de concevoir qu'un genre à caractère fortement interactif, comme l'entretien, peut non seulement comporter des segments hétérogènes, mais aussi des segments (types de discours) à caractère non interactif, comme le discours théorique. Et si la production de ce type de discours porte bien entendu l'empreinte de la production orale, il n'en demeure pas moins que 
l'attitude énonciative sous-jacente est liée à la tentative du locuteur de s'extraire des contingences d'un contexte particulier; et ce mouvement revêt une importance considérable pour l'interprétation de l'agir, ouvrant la voie à des processus de généralisation, comme en témoigne la construction des " figures d'action canonique et définition », dont il sera question dans la partie 4 de cet article.

\subsubsection{L'analyse des repérages temporels}

Les types de discours se caractérisent aussi par une relative spécificité de leur organisation temporelle (voir Bronckart, 1997, chapitre 7). Les opérations concernées font intervenir trois paramètres : le moment de l'acte de production, le moment présumé de l'action ou de l'état verbalisé dans le texte, et les axes de référence temporelle qui sont créés dans et par la production textuelle. Ces axes de référence peuvent être bornés, ou d'une durée limitée (dans ce cas, ils peuvent être explicités par des unités comme aujourd'hui, hier, ce mois-ci, etc.) ou non bornés et de durée illimitée (dans ce cas, ils peuvent être explicités par des unités comme habituellement, généralement, etc.). Les opérations de repérage temporel consistent à mettre en rapport le moment de l'action verbalisée, soit avec le moment de l'acte de production, soit avec l'axe de référence temporelle. Et ces mises en rapport peuvent être de l'ordre de l'inclusion, de la simultanéité, de l'antériorité et de la postériorité. Dans les trois types de discours qui apparaissent dans les textes du genre «entretien », les principaux mécanismes de repérage temporel sont les suivants.

a. Pour le discours interactif :

- création d'un axe de référence temporelle borné, construit par extension psychologique du moment de l'acte de production ; dans ce cas, le repérage des états ou actions verbalisés se réalise par établissement d'un rapport de simultanéité, de postériorité ou d'antériorité eu égard à l'axe de référence.

- création d'un axe de référence non borné ; dans ce cas, le repérage des états ou actions verbalisés se réalise par inclusion au sein de cet axe.

b. Pour le discours théorique :

- création d'un axe de référence temporelle non borné, implicite ou explicité ;

- $\quad$ repérage des états ou actions verbalisés par inclusion au sein de cet axe ;

- non-pertinence du moment de l'acte de production.

c. Pour le récit interactif :

- création d'un axe de référence temporelle borné, situé en amont de la situation d'interaction, et généralement explicité (hier, il y a deux jours, etc.) ;

- repérage des états ou actions verbalisés, soit par inclusion au sein de cet axe, soit par un rapport d'antériorité eu égard au moment de l'acte de production.

\subsubsection{L'analyse des modes d'expression de l'agentivité}

Cette analyse vise à identifier les différents modes d'expression des personnes impliquées dans l'activité de travail concernée, ainsi que les modes d'expression d'autres éléments ayant une importance pour le déroulement de cette activité. Lorsque le sujet d'une phrase est le travailleur concerné par l'agir, on relève si ce travailleur est désigné par une des formes pronominales possibles (je, tu, on, nous, $\square$ ), ou par un le nom générique de sa profession (par exemple l'infirmière, l'enseignant-e), ou par un nom propre (par exemple, Sylvie), etc. Et l'on procède à une analyse analogue lorsque le sujet de la phrase est un humain protagoniste de la situation de travail, ou encore un élément qui est important dans cette même situation (un instrument par exemple).

A titre d'exemple, voici un relevé des modes d'expression de l'agentivité des enseignants, adapté à partir de celui initialement proposé pour le corpus d'entretiens avec les infirmières (et adaptable encore selon les caractéristiques effectives des données).

a. Enseignant

Enseignant(e) seul(e) $(j e, t u, \varnothing)$

Enseignant(e) collectivisé(e) (on, nous)

Autre enseignant individuel ( $\mathrm{il}$, elle, nom propre ou nom commun, p. ex. ma collègue) 
Autres enseignants collectifs (ils, noms propres ou communs)

b. Complexe enseignant / élève(s) indifférencié (on, nous)

c. Elève

Individuel ( $i l$, elle, nom propre ou nom commun, p. ex. le gamin, l'étudiant)

Collectif (ils, noms propres ou communs)

d. Instrument (matériel relatif à l'enseignement : noms)

e. Impersonnel (il, ce, ça)

\subsubsection{L'analyse des relations prédicatives indirectes}

Cette analyse concerne les relations entre le sujet et le verbe des phrases/énoncés, et elle a consisté d'abord à distinguer les relations prédicatives directes (par exemple je fais; je donne) et les relations prédicatives indirectes, c'est-à-dire dans lesquelles un semi-auxilaire, ou métaverbe, est introduit entre le

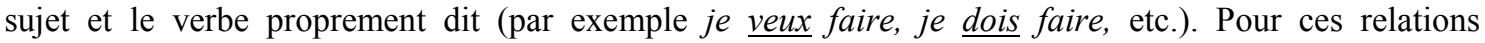
indirectes, l'analyse a visé à établir les valeurs introduites par les métaverbes, à savoir :

a. les valeurs de modalisation

- épistémique (ordre du possible, du certain, etc.);

- déontique (ordre de l'obligation sociale, morale);

- appréciative (ordre du ressenti subjectif en termes de beau, bien, etc.);

- pragmatique (ordre des capacités, intentions, volonté de l'actant);

b. les valeurs aspectuelles (exprimées par les métaverbes du type commencer à, finir de, être en train de, etc.) ;

c. les valeurs d'opération psychologique (exprimées par les métaverbes du type penser, croire, etc.).

Cette analyse est complétée par l'identification et le classement de marques de modalisation qui ne sont pas intercalées dans la relation prédicative (sans doute, peut-être, certainement, heureusement, malheureusement, etc.).

\section{Les caractéristiques du discours des enseignants et des « figures d'actions » qu'ils produisent}

Les entretiens ont été analysés selon la méthodologie qui vient d'être décrite, cette analyse étant centrée, dans un premier temps, sur l'interprétation de l'agir enseignant de manière générale, c'est-à-dire en amont d'un regard spécifiquement didactique. Les résultats obtenus ré-exploitent la notion de "figures d'action » (voir Bulea, 2007, 2009 ; 2010 ; Bulea \& Bronckart, 2010), initialement développée sur la base des entretiens avec des infirmières, mais qui s'est avérée tout à fait pertinente — au prix de certaines extensions/adaptations, on le verra — pour l'analyse des textes portant sur d'autres types d'agir professionnel.

Les «figures d'action» sont des configurations discursives, correspondant à autant de formes d'interprétation de l'agir, qui reposent notamment sur l'exploitation alternée, dans le cadre du même entretien, de différents types de discours (discours interactif, discours théorique et récit en particulier) et de différents axes temporels de référence (axe de la situation d'interaction, axe situé en amont ou en aval de cette situation, ou encore non borné), ainsi que sur la mobilisation différenciée de noms et pronoms codifiant les actants, ou encore de mécanismes énonciatifs, dont en particulier les modalisations et les prises en charge énonciatives. Au nombre de cinq, ces « figures d'action » présentent les caractéristiques générales qui suivent.

La figure de l'action occurrence constitue une saisie de l'agir caractérisée par un très fort degré de contextualisation. Sa construction repose sur l'identification d'un ensemble d'ingrédients de l'agir saisis 
dans leurs dimensions particulières, spécifiques (tel enseignant, telle classe, tels élèves, tel contexte, etc.). Cette figure apparaît quasi exclusivement dans des segments de discours interactif, structurés selon un axe de référence temporelle qui est celui de la situation d'interaction; néanmoins, des axes de référence locaux peuvent apparaitre, ces derniers constituant souvent des projections vers le moment (ou une phase particulière) de l'agir. De même, on remarque de nombreuses occurrences de discours rapporté. Du point de vue des marques d'agentivité, l'actant est désignée par des «je », ce qui signale sa forte implication, ou son statut d'acteur ${ }^{6}$. Enfin, cette figure se caractérise par un nombre relativement important de relations prédicatives indirectes, notamment des modalisations pragmatiques (voire le début de l'exemple 1b).

(1a et1b) Exemples de figure de l'action occurrence ${ }^{7}$

...ben $t u$ vas leur présenter les objectifs de façon extrêmement simple donc tu vas écrire au tableau aujourd'hui on va apprendre à parler au passé / parler d'hier $\uparrow$ / pour ça tu vas leur dire quelle date on est aujourd'hui $\downarrow$ i'vont te dire la date avec les chiffres [INT : hum hum] et après tu vas leur demander la date d'hier $\uparrow /$ juste pour les mettre en confiance et leur dire dans quel espace temps on s'trouve [INT: d'accord] après ça et bien tu vas par exemple prendre des exemples de leur vie à eux (Entretien avec RV, I1, partie instruction au sosie)

...si tu veux tu peux prendre des cartes [INT: oui] avec des verbes par exemple [INT : oui] ou avec des dessins [INT : oui] et puis leur demander soit ce qui s'est passé / soit euh:: / euh / si tu montres des dessins à / dans une situation qu'est-ce qui s'est passé avant $\uparrow$ (Entretien avec HT, I1, partie instruction au sosie)

La figure de l'action événement passé propose une saisie rétrospective de l'agir, dans laquelle ce dernier est toujours saisi dans sa singularité, mais sans rapport de contiguïté avec la situation de sa mise en forme langagière. Cette figure consiste en la délimitation et en l'extraction du passé d'une unité praxéologique identifiée, saillante et illustrative de l'agir, et elle témoigne d'une contextualisation manifeste, mais fragmentaire et sélective. Elle apparaît dans des segments de récit interactif, les actes évoqués étant saisis en référence à un axe temporel situé en amont de la situation d'entretien, et dont l'origine est marquée (par exemple, hier, il y a trois jours, la dernière fois, une fois, etc.). L'actant demeure impliqué dans l'événement raconté, ce qui est marqué par la présence du pronom « je ». Cette figure se caractérise enfin par une structuration des faits racontés potentiellement proche du schéma narratif (situation initiale, complication, résolution, évaluation), qui confère à l'unité extraite son statut d' "événement ».

(2) Exemple de figure de l'action événement passé

...il m'est arrivé d'avoir des difficultés (...) quand t'as deux nationalités opposées :: /

euh j'ai eu une fois un Palestinien et puis un :: / un Israelien / ben ils se sont levés /

ils étaient prêts à se taper l'un sur l'autre / pis faut gérer ça quoi/ [INT : xxx] $j$ 'tape sur la table / $j$ 'tape sur la table aussi hein / en disant que ça s'passe pas xxx même pas à l'intérieur de la classe (Entretien avec HT, I1, partie semi-directive)

La figure de l'action expérience constitue une saisie de l'activité sous l'angle de la cristallisation personnelle de multiples occurrences d'activités vécues : elle propose une sorte de bilan de l'état actuel de l'expérience de l'actant eu égard à la tâche concernée. Elle apparaît principalement dans des segments de discours interactif, mais avec un axe de référence temporelle non borné, marqué par des adverbes et locutions adverbiales à valeur généralisante (normalement, toujours, de toute façon, etc.). Son organisation discursive tend à reproduire l'ordre chronologique de l'activité, mais signale aussi des points de bifurcation de celle-ci, ainsi que des adaptations mises place par l'actant (formulations en si...alors).

(3) Exemple de figure de l'action expérience ...par exemple pour les traducteurs si j'ai des traducteurs dans un groupe euh je travaille parfois un texte mais au lieu de faire seulement de la compréhension:: globale [INT : ouais] et de la discussion:: [INT : ouais] euh s'il y a des traducteurs dans le groupe je vais m'adapter et faire proposer parallèlement peut-être facultativement ils peuvent le faire à la maison un travail de vocabulaire ou dans les mots soulignés dans le texte ils doivent trouver des synonymes qui sont proposés 
dans :: / en annexe [INT: d'accord] donc je m'adapte un peu surtout pour les traducteurs (Entretien avec GL, I2, partie semi-directive)

La figure de l'action canonique réside en une saisie de l'activité sous forme de construction théorique, et elle propose une logique de la tâche présentée comme a-contextualisée, à validité générale. De ce fait, elle rend compte surtout de la structure chrono-logique prototypique de l'agir, ainsi que des normes qui le régissent. Cette figure s'organise sous forme de discours théorique, ou de mixte théorique-interactif, qui organise une suite de verbes conjugués au présent générique, évoquant des actes dont l'ordre tend à reproduire la chronologie générale de l'agir. Cet ordre est en outre exprimé au travers d'une organisation phrastique canonique, du type sujet - verbe - complément, qui comporte souvent des modalisations déontiques (il faut, on doit, etc.) ainsi que des marques agentives (on, tu impersonnel) attestant d'une très faible, voire inexistante, actorialisation de l'enseignant.

(4a et $4 \mathrm{~b})$ Exemples de figure de l'action canonique

...on a un programme et / on a une liste de matériel à utiliser qu'on doit utiliser / la

façon dont tu / on l'utilise n'a aucune importance mais / le matériel doit être utilisé

[INT : xxx] exploité (Entretien avec VH, I2, partie semi-directive)

...à l'Ixxx (nom de l'institution) c'est différent (petit rire) /// [INT : de quelle façon / par exemple (voix très basse, sur le ton de la confidence)] euh j'veux dire tu suis la méthode / tu remets pas en question / les étudiants doivent suivre (Entretien avec HT, I1, partie semi-directive)

La figure de l'action définition relève d'une saisie de l'activité en tant qu'objet de réflexion. Contrairement aux autres figures, elle ne thématise ni les actants, ni l'organisation chronologique de l'activité (d'où la quasi absence de pronoms codifiant l'enseignant comme sujet de verbes exprimant des actes d'enseignement), mais rassemble des traits jugés pertinents, susceptibles de la circonscrire et de la différencier d'autres sortes d'activités. Elle est insérée dans des segments relevant du discours théorique, mais contrairement à l'action canonique, les formes verbales mobilisées ne portent que rarement sur des actes ou des gestes. La grande majorité des relations prédicatives est constituée de constructions impersonnelles en c'est et il y a. Si, par rapport aux autres figures, l'agentivité de l'enseignant est ici quasi nulle, elle est par contre très fortement marquée du point de vue énonciatif, l'action définition étant la figure qui comporte, proportionnellement, le plus grand nombre de mécanismes de prise en charge énonciative. La responsabilité ou l'actorialité de l'enseignant se trouve donc ici transférée en quelque sorte sur l'acte même de « dire le travail » : presque toutes les occurrences d'action définition comportent le marqueur d'identité je (voir l'exemple 5 ci-dessous, qui commence par je sais que et se termine par je dirais) qui marque la voix propre de l'enseignant, prenant en charge ce qui est dit.

(5) Exemple de figure de l'action définition

... je sais que je ne peux pas arriver non préparée à un cours par exemple c'est pas me remettre en question mais c'est c'est d'être toujours au moins au courant de tout ce qui se passe en Euro::pe au sujet des :: des :: / ben des réformes et des choses comme ça (...) donc euh oui c'est / c'est d'être toujours ouais sur le fil de l'actualité je dirais (Entretien avec $\mathrm{VH}, \mathrm{I} 2$, partie semi-directive)

Pour intéressante qu'elle soit - y compris en ce qu'elle constitue un moyen de comparaison entre la saisie de l'agir par les travailleurs d'une part, dans des textes qu'ils produisent, et par les concepteurs du travail d'autre part, dans des textes institutionnles et/ou de prescription - la construction des figures d'action n'est pas en soi caractéristique du discours enseignant. Si l'on fait abstraction notamment du lexique propre à la profession (cours, étudiants, classe, programme, compréhension, texte, vocabulaire, synonymes, apprendre à parler au passé, écrire au tableau, etc.), ce processus morphogénétique est discursivement similaire à celui mis en place par les infirmières, les figures d'action que celles-ci produisent mobilisant des opérations et des ressources énonciatives proches, voire identiques, à celles mobilisées par les enseignats. A titre d'exemple, voici trois extraits, issus d'entretiens recueillis lors de la recherche sur le travail infirmier, et relevant des figures d'action événement passé, expérience et canonique : 
(6) Exemple de figure d'action événement passé, travail infirmier

...la dernière fois par exemple tu vois je devais enlever des fils à un patient / et:: // il avait / une collection de / de liquide séreux au niveau de la cicatrice j'ai la cicatrice qui s'est ouvert // le bout de la cicatrice / il avait une médiane / c'était le bout de la cicatrice qui s'est ouvert / tu vois [INT : au moment où tu as fait le pansement] où j'ai enlevé les fils et puis tu sais j'ai commencé à appuyer et puis ça coulait / et heu:: ça s'est ouvert tu vois / pis dans ce cas-là ben t'appelles l'opérateur et puis tu lui demandes ce qu'il veut / ce qu'il veut faire (Entretien avec $\mathrm{N}$ - chirurgie digestive)

(7) Exemple de figure d'action expérience, travail infirmier

...bon / j'fonctionne toujours comme ça / je vois si le patient est disponible dans la matinée s'y a pas d'examen s'y a pas de chose comme ça / j'préviens le patient qu'on va faire son pansement // des fois ça peut être dix minutes avant / des fois ça peut être une demie heure une heure // si c'est quelqu'un très angoissé on évite de le prévenir trop avant parce que sinon (rires) (Entretien avec $\mathrm{S}$ - chirurgie digestive)

(8) Exemple de figure d'action canonique, travail infirmier

...le soin il commence au moment où on rentre dans la chambre (...) l'installation heu on déballe la plaie on prépare le / le set [INT : hum hum] / après on fait le pansement on communique / on ferme le pansement on réinstalle le patient on met la ceinture on réhabille / on remet bien le lit en position on lui propose d'aller au fauteuil s'il doit aller au fauteuil ou il reste comme ça on lui remet tout son matériel à disposition / on tire le rideau et après / on on dit heu / voilà / au revoir au patient (Entretien avec $\mathrm{S}$ chirurgie digestive)

Le lexique est, dans le cas des infirmières, propre à leur profession et à leur milieu de travail (cicatrice, collection de liquide, médiane, pansement, patient, lit, rideau, déballer une plaie, appeler l'opérateur, etc.). Mais au plan de l'organisation discursive, un ensemble de similitudes peuvent être observées : ainsi, s'agissant de l'action événement passé, la saisie rétrospective de l'agir (ici un pansement abdominal postopératoire) mobilise, comme chez les enseignants, le récit interactif, un axe temporel de référence situé en amont de la situation d'interaction et marqué (la dernière fois), ainsi que les mécanismes d'implication de l'actant, implication marquée par la présence du pronom je. En ce qui concerne l'action expérience, on constate toujours la mobilisation d'un axe de référence non borné (marqué par je fonctionne toujours comme ça) à l'intérieur du discours interactif, de même que l'expression de bifurcations possibles et relativement régulières dans le cours de l'agir (si, des fois). Enfin, quant à la figure de l'action canonique, la mobilisation du discours théorique ou du mixte théorique-interactif est accompagnée de la structuration phrastique récurrente sujet - verbe - complément, ainsi que d'un très net affaiblissement de l'actorialité du professionnel, marqué par la présence massive du pronom on.

La spécificité de l'interprétation de l'agir par les enseignants doit ainsi être recherchée ailleurs que dans la teneur énonciative générale des figures produites : il s'agit du fait que, conjointement à la production des cinq figures d'action qui viennent d'être décrites (avec le lexique de la profession), les enseignants tendent à substituer à l'analyse de leur propre travail, ou à combiner intimement à cette analyse, une description et un commentaire de l'agir de leurs élèves, ou étudiants. Suivant des propositions formulées ailleurs (voir Bulea \& Bronckart, 2012), on peut dès lors différencier les figures d'action internes, dont le référent est l'agir spécifique de la personne interviewée (l'enseignant, comme dans les exemples cidessus), et les figures d'action externes, dont le référent est l'agir d'autres protagonistes de la situation de travail, en particulier les élèves ${ }^{8}$. Les propriétés linguistiques des figures d'action externes sont globalement les mêmes que celles des figures d'action internes correspondantes, mais il existe une différence dans le domaine de la cohésion nominale, dans la mesure où ces figures comportent des séries isotopiques dont l'origine est exprimée par une forme nominale codifiant les apprenants et les reprises anaphoriques sont constituées de pronoms à la troisième personne (il, elle, ils, etc.).

En réalité, les entretiens réalisés avec les enseignants se caractérisent par un va-et-vient permanent, ou une alternance, ou encore une forme d'articulation, entre des figures d'action internes et externes; et cette alternance - qui est quasi absente dans le cas d'autres métiers, comme celui d'infirmière ou d'opérateur 
en entreprise - est présente aussi bien dans la partie «entretien semi-directif » que dans la partie « instruction au sosie ». En voici deux exemples, relevant de chacune de ces parties ${ }^{9}$ :

(9) Enchaînement action occurrence externe et action occurrrence interne

... donc ils voient euh déjà:: que quelque chose se passe mais ils savent pas quoi / eux

ils parlent de leur semaine de travail [INT: d'accord] et c'est sous forme de discussion de tour de table / toi [INT : d'accord] tu notes [INT : d'accord] au fur et à mesure / et puis quand ils ont dit le maximun:: euh ou quand ton tableau est rempli [INT : ouais] hein euh tu dis et ben voilà en général voilà ce que vous faites pendant la semaine / donc en bleu toi par exemple tu reprends un peu ce que $\mathrm{X}$ ou $\mathrm{Y}$ a dit / toi tu dis tu travailles de telle heure à telle heure [INT : oui] hein / et cette semaine qui vient de se passer est-ce que tu as travaillé:: de telle heure à telle heure (Entretien avec GL, I2, partie instruction au sosie)

(10) Enchaînement action définition externe et action définition interne ...oh à Fxxx (nom de l'institution) c'est [ne] pas savoir apprendre / c'est [ne] pas forcément avoir l'intérêt d'apprendre / préférer rester à la maison / en ayant leurs indemnités chômage / euh (2s) oui puis c'est peu de scolarité donc comment leur apprendre à :: à apprendre s'ils veulent le faire aussi / et puis c'est respecter leurs douleurs quand ils ont des douleurs etc c'est pas facile aussi de devoir travailler en se disant ben il a pas la tête ici pour une raison ou une autre (Entretien avec HT, I1, partie semi-directive)

Ce dédoublement de figures d'action nous paraît être lié à trois facteurs au moins. Premièrement, cela montre que, dans le cadre de la profession enseignante, l'interprétation de l'agir propre est quasi indissociable de celle de l'agir des élèves, comme si une saisie exclusive du premier était inadéquate par rapport aux propriétés générales de l'activité ; et ce phénomène ne nous paraît pas sans lien avec une des caractéristiques du « discours enseignant ambiant », mise en évidence par Bronckart \& Machado (2005) : dans les textes institutionnels du domaine scolaire, sont systématiquement thématisés les élèves en tant que bénéficiaires de l'agir enseignant et l'effet escompté de cet agir, et ce plus que l'actorialité même des enseignants.

Deuxièmement, les figures d'action externes fournissent aux enseignants une possibilité d'interprétation des résultats de l'agir propre, sous une forme non pas déclarative, globalisante et statique, mais dynamique, potentiellement progressive, et située à l'intérieur même de l'activité collective. La construction des figures d'action externes est une occasion de prise de conscience, de la part des enseignants, de ce que leur faire produit comme agir, comme processualité chez les élèves, et non seulement comme état, ou nouveau stade, de maitrise des connaissances.

Troisièmement, les figures d'action sont potentiellement des sources d'évaluation les unes pour les autres : ainsi, l'interprétation de ce que les élèves font (ou ne font pas, ou font difficilement, etc.) au travers de la construction des figures d'action externes peut conduire à une réévaluation de certaines dimensions de l'agir propre, saisies, elles, par les figures d'action internes, de manière rétrospective, prospective ou généralisante.

\section{Comment les « figures d'action » saisissent-elles les dimensions didactiques de l'activité ?}

En ré-examinant les figures d'action produites par les enseignants de notre recherche sous l'angle spécifique des dimensions didactiques qui y sont attestables, nous organiserons nos observations en quatre points.

1. La dimension la plus autonome, dont l'évocation semble indépendante de l'une ou l'autre des figures d'action, est celle du matériel pédagogique et des moyens d'enseignement. Cette dimension peut faire l'objet tantôt d'une thématisation générique, non incluse dans la construction de figures (exemple 11, 
infra), tantôt d'une saisie contextuelle, incluse dans le cadre de l'action occurrence (exemple 12), ou encore d'une saisie en rapport avec des normes de travail, à l'intérieur de l'action canonique (voir l'exemple 4a, que nous reproduisons ci-dessous).

(11) Thématisation du matériel hors figures d'action

...il y a effectivement / disons qu'au niveau du matériel on a beaucoup de / beaucoup de supports (rires) / de médias qu'on peut utiliser / soit la télévision / les rétroprojecteurs / on a aussi des / on peut aussi utiliser des ordinateurs, mais euh / c'est pas très en phase avec ce qu'on fait en tant qu'enseignant de langues / mais il y $a$ des possibilités / euh / on $a$ des supports pédagogiques qui sont faits en fonction des /des publics et des niveaux donc oui il y a:*. (Entretien avec MB, I1, partie semidirective)

(12) Thématisation du matériel à l'intérieur de l'action occurrence

...si tu veux tu peux prendre des cartes [INT: oui] avec des verbes par exemple [INT : oui] ou avec des dessins [INT : oui] et puis leur demander soit ce qui s'est passé / soit euh:: / euh / si tu montres des dessins à / dans une situation qu'est-ce qui s'est passé avant $\uparrow$ (Entretien avec HT, I1, partie instruction au sosie)

(4a) Thématisation du matériel à l'intérieur de l'action canonique

...on a un programme et / on a une liste de matériel à utiliser qu'on doit utiliser / la façon dont $t u$ / on l'utilise n'a aucune importance mais / le matériel doit être utilisé [INT : xxx] exploité (Entretien avec VH, I2, partie semi-directive)

On observe en outre que lorsque les moyens pédagogiques sont évoqués hors figures d'action, ceux-ci sont saisis tendanciellement dans leur matérialité "première », c'est-à-dire en tant que supports ou artefacts indépendants d'une pratique particulière, à disposition des enseignants (voir Plane et Schneuwly, 2000, pour une analyse générale du statut des outils / instruments / moyens d'enseignement). De ce fait, et dans le ce type de saisie, les moyens pédagogiques sont situés en quelque sorte en amont de leur rôle dans la création de «milieux didactiques » ou d'apprentissage (Brousseau, 1988), étant simplement des ressources potentielles, à l'égard desquelles l'enseignant met en place une démarche de tri ou de choix, comme il ressort de l'extrait 11 ci-dessus. Notons l'abondance, dans les extraits de ce type, d'expressions mobilisant le verbe « avoir », alternant avec celles mobilisant les verbe « utiliser » (dans l'exemple11, les expressions il y $a$, on a reviennent à plusieurs reprises, en alternance avec on peut utiliser). L'action canonique semble propice à une interprétation analogue, mais elle offre davantage un cadre de perception de ces moyens en tant que faisant l'objet d'une prescription institutionnelle et non pas d'un choix individuel de l'enseignant, comme il ressort de l'exemple 4 a (voir les modalisations du verbe utiliser : on peut utiliser attestable en 11 laisse la place à on doit utiliser, le matériel doit être utilisé).

En revanche, lorsque les moyens pédagogiques sont saisis à l'intérieur de la figure d'action occurrence, leur fonction dans la création de milieux didactiques apparaît plus nettement, comme il ressort de l'exemple 12. L'évocation de ces moyens s'accompagne d'ailleurs d'une bien plus grande variété de verbes, codifiant des actes de l'enseignant lors de la leçon ou du cours : dans l'extrait 12, par exemple, prendre des cartes, leur demander, montrer des dessins. Ce même phénomène est attestable dans l'extrait qui suit, provenant d'un entretien avec une enseignante de l'institution I2 (l'exemple 12 relève de I1). Le matériel pédagogique, des images, est clairement saisi en tant que contribuant à la mise en place d'un milieu propice à l'apprentissage, ici à une démarche inductive (tu acceptes toutes les réponses même si elles sont incomplètes, tu les amènes à remarquer, etc.), les actes et gestes de l'enseignant étant tout aussi clairement décrits (tu leur apportes des images, tu les mets au tableau, tu les mets au rétroprojecteur, tu fais une photocopie par étudiant, tu poses la question, tu acceptes toutes les réponses, etc.) :

(13) ... tu leur apportes des images [INT : de images de quoi] ben par exemple d'un ho::mme avec l'allure euh relativement juvénile seul euh sans chien sans chat sans famille sans enfant sans rien [INT : hum hum] le même homme euh un p'tit peu plus tard avec un gros ventre grosse maison grosse famille et que s'est-il passé entre les deux / les images tu les mets au tableau / tu les mets au rétroprojecteur en fonction des appareils que t'as [INT: d'accord] à ta disposition / tu fais une photocopie par étudiant [INT : d'accord] en fonction de ton groupe [INT : donc je leur donne des 


\begin{abstract}
images et ils doivent me produire des phrases] voilà / tu / tu poses la question que s'est-il passé et euh:: tu acceptes toutes les réponses même si elles sont incomplètes pour commencer euh avec des phrases un peu euh ben un peu branlantes c'est pas le problème (...) tu les:: amènes à:: remarquer qu'on a utilisé être ou avoir [INT : ouais] euh:: et puis t'essaies de leur faire construire une théorie (Entretien avec LH, I2, partie instruction au sosie)
\end{abstract}

Ainsi, si le matériel pédagogique est la dimension didactique la plus autonome, au sens où il peut apparaître en dehors ou à l'intérieur des figures d'action, le dévoilement de sa fonction instrumentale proprement dite, de son implication tant dans la transformation possible des connaissances des apprenants, que dans l'intention transformatrice de l'enseignant, semble intimement lié à la construction de certaines figures d'action, notamment de l'action occurrence (comme en témoignent les exemples analysés ici), mais aussi de l'action expérience ou, plus rarement, de l'action événement passé.

2. La problématique de l'objet enseigné semble quant à elle apparaître de manière préférentielle dans le cadre de la figure de l'action occurrence, ce qui montre une certaine difficulté de saisir cette dimension didactique autrement que de manière contextualisée, et en relation avec certains gestes didactiques, notamment la présentification, la décomposition et le pointage. Ainsi, tous les extraits d'instruction au sosie comportent en fait de très longs passages ou les enseignants déploient un vrai «scénario didactique », comme il ressort de l'extrait ci-dessous (drastiquement abrégé néanmoins).

\begin{abstract}
(14) Thématisation de l'objet et de gestes didactiques dans l'action occurrence ....après tu vas leur demander la date d'hier (...) après ça et bien tu vas par exemple prendre des exemples de leur vie à eux (...) après et ben tu vas leur expliquer comment ça marche / donc tu vas leur réexpliquer le verbe avoir qu'ils connaissent déjà (...) tu vas utiliser des fiches de couleur / tu vas écrire le verbe au tableau / tu vas enlever la fiche et et tu vas la remplacer par la fiche é et tu recommences à chaque fois pour chaque exemple que tu fais (...) après tu vas l'faire à l'oral / tu vas donner des verbes qu'ils connaissent déjà / parler / danser/ chanter / étudier / et leur demander de faire des phrases / ils vont inventer / qu'est-ce que tu as fait hier [INT : d'accord ] et après on va les écrire... (Entretien avec MB, I1, partie instruction au sosie)
\end{abstract}

S'agissant de la problématique de l'objet enseigné, explicitons ici le fait que le choix du passé composé en tant que thème de l'instruction au sosie a présenté un caractère quasi aléatoire : il n'était motivé que par sa transversalité supposée, par l'hypothèse que l'enseignement du passé composé pouvait a priori concerner tous les enseignants et tous les publics apprenants des institutions où se déroulait notre recherche ; et que donc les enseignants interviewés étaient susceptibles d'entrer plus facilement dans la posture requise par la technique de l'instruction au sosie. A posteriori, on peut remarquer que cette hypothèse ne s'est vérifiée que partiellement: contrairement aux enseignants des deux premières institutions, qui sont très facilement entrés dans le thème, les enseignants de la troisième institution (universitaire) ont initialement exprimé leur orientation vers un enseignement plus nettement textuel que grammatical, et ont de ce fait procédé à une reconstruction de l'objet initialement proposé par l'intermédiaire de la composante textuelle évoquée. En voici un exemple :
(15) ...bon j'vais prendre un simple / donc euh un cours assez simple [...] qui s'adresse au public de l'année propédeutique [INT : hum hum] auquel je donne un cours le vendredi de dix heures à midi / et qui doit en fait apprendre à raconter oralement un conte de son / généralement de préférence de sa culture d'origine [INT : hum hum] et puis ça c'est la moitié du cours où les étudiants racontent et puis l'autre moitié c'est moi qui leur propose un texte sur lequel on / on travaille et près qu'ils se racontent entre eux (Entretien avec NB, I3, entrée dans l'instruction au sosie)

Mais une fois cette réorientation effectuée, la saisie de l'objet enseigné, du point de vue des liens qui se tissent entre ce dernier, le contexte effectif d'enseignement et les gestes didactiques dans une sorte de scénario, semble se réaliser de manière analogue, tendanciellement toujours au sein de la figure de l'action occurrence. En voici un exemple : 


\begin{abstract}
(16) ... vous vous attribuez à chacun une espèce de rôle d'écoutant actif [INT : hum hum] et puis après la personne raconte et vous vous prenez des notes sur les erreurs de français et puis en essayant de prendre des notes sur ces différentes rubriques (...) donc la personne qui est responsable de la situation initiale fait un commentaire (...) et une fois que la personne a parlé vous / vous corrigez / vous dites si vous êtes d'accord ou pas [...] et donc on fait un petit tour de table et puis voilà / après on distribue le document (...) et puis on retourne les feuilles et puis je demande... (Entretien avec NB, I3, suite de l'instruction au sosie)
\end{abstract}

Néanmoins, et au-delà du constat de la mise en œuvre de ces liens et de leur saisie dans et par la construction de l'action occurrence, des analyses complémentaires devront être effectuées pour décrire plus finement la teneur interne de ces scénarios, afin de mieux cerner la place qu'occupe l'objet enseigné et ses particularités dans la construction de ceux-ci.

3. Comme cela a été largement documenté sur la base d'analyses des activités des enseignants en classe (voir notamment Sensevy, 2005 ; Sensevy, Mercier \& Schubauer-Leoni, 2000 ; Aeby-Daghé \& Dolz, 2008 ; Schneuwly, 2009), à l'avènement de l'objet enseigné concourent un ensemble de gestes didactiques, parmi lesquels, et sans souci d'exhaustivité ${ }^{10}$, on mentionnera : la mise en place de dispositifs didactiques, notamment par l'intermédiaire des consignes; - la présentification, qui consiste à rendre l'objet présent, à le montrer aux étudiants en utilisant des moyens adéquats ; - la décomposition, qui implique une hiérarchisation des dimensions de l'objet, et qui se traduit par la mise en place d'une séquence d'enseignement; - le pointage, qui consiste à focaliser l'objet ou une de ses dimensions ; - la construction de la mémoire didactique, qui vise la recomposition de ce qui a été décomposé, et qui se traduit notamment par l'appel aux connaissances préalables et par des effets d'annonce de ce qui va être abordé ; - l'institutionnalisation, qui réside en la «fixation explicite et conventionnelle du statut d'un savoir pour construire un apprentissage » (Sensevy, cité par Aeby-Daghé \& Dolz, op. cit., p. 86), et qui permet la prise de conscience de ce que l'objet enseigné fait partie d'une culture disciplinaire, étant de ce fait transférable et exigible dans d'autres contextes.

Si les gestes de présentification, décomposition et pointage semblent préférentiellement associés à la saisie de l'objet enseigné dans l'action occurrence (voir point 2 ci-dessus), le geste d'institutionnalisation semble quant à lui faire l'objet d'une saisie tendanciellement à l'intérieur de l'action canonique et de l'action définition, intégrant de ce fait cette « logique générale » de l'agir que la première figure d'action construit.

(17) Institutionnalisation dans l'action canonique et définition interne

...et puis après on fait le transfert au livre à la grammaire progressive du français

voyez c'est ça / les verbes réguliers c'est telle page / les verbes irréguliers // bon

(Entretien avec GL, I2, partie instruction au sosie)

De manière intéressante, ce geste est parfois dévolu aux apprenants, les enseignants mettant en scène une forme de prise de conscience du statut du savoir par ceux-ci, au travers de la mobilisation du discours rapporté (maintenant je sais que ça c'est le passé composé...), comme il ressort de l'extrait suivant, qui se situe en parfaite continuité du précédent.

(18) Institutionnalisation dans l'action canonique externe, projetée sur les étudiants ...ils aiment bien voir quand mê::me quelque chose pour fixer /[INT: d'accord] donc maintenant je sais que ça c'est le passé composé / je sais où ça se trouve dans ma grammaire et je peux / peut-être faire un exercice récapitulatif où il y a pas seulement les verbes réguliers (Entretien avec GL, I2, partie instruction au sosie)

4. Dans la mesure où l'action occurrence est la figure la plus permissive, comportant le plus de dimensions didactiques, le geste de mise en place de dispositifs didactiques y apparaît régulièrement (voir l'exemple 1, supra); mais ce geste semble par ailleurs être au cœur et à l'origine des moments, dans l'instruction au sosie, où l'enseignant quitte le «tu» imposé par la consigne de cette technique pour « récupérer » l'agentivité exprimée à la première personne («je »), conjointement à la possibilité de faire état de ses routines ou de ses préférences dans le choix des tâches et dans la conduite de l'activité. Ce 
passage du «tu » à « je » mobilise souvent des structures emphatiques, et correspond aussi au passage de l'action occurrence à l'action expérience, comme il ressort des extraits suivants.

$(19 \mathrm{a}, 19 \mathrm{~b}, 19 \mathrm{c})$ Retour à la première personne et saisie de la mise en place du dispositif didactique

...si tu vois qu'il y a des difficultés tu peux éventuellement t'arrêter / discuter/ c'que
j'aime bien faire aussi c'est d'leur dire de s'aider mutuellement donc de faire souvent

l'exercice à deux (Entretien avec MB, I1, partie instruction au sosie).

...tu les laisse parler et tu retranscris tout / que tu montres / ou ils le remarquent par eux-mêmes que / y'a des participes passés qui changent / [INT : ouais ] ensuite en général / je :: euh / j'efface tout / [INT : oui] j'leur fait une petite dictée puisque à Fxxx (nom de l'institution) euh on travaille beaucoup les dictées $\downarrow /$ donc une dictée au passé composé (Entretien avec HT, I1, partie instruction au sosie)

\begin{abstract}
...tu acceptes toutes les réponses même si elles sont incomplètes pour commencer euh avec des phrases un peu euh :: ben un peu branlantes c'est pas l'problème [S : donc je note les phrases] tu les répètes / moi je procède comme ça je les répète et ensuite au tableau je ne mets que des phrases correctes mais qui viennent d'eux (Entretien avec LH, I2, partie instruction au sosie)
\end{abstract}

Plusieurs questions encore auraient, bien entendu, mérité d'être posées, qui concernent, d'une part, l'analyse plus fine, et conduite dans une perspective comparative (selon les parties du dispositif d'analyse de l'activité, les types de public, l'expérience des formateurs, etc.), de la manière dont les dimensions didactiques de l'activité enseignante sont thématisées à l'occasion de l'interprétation de l'activité ; d'autre part, l'exploitation que l'on peut faire de ce type de travaux dans le cadre de la formation des enseignants. Ces questions constituent autant de pistes pour la poursuite de ce travail, qui nous aura néanmoins permis de constater que l'interprétation de l'agir enseignant repose, in fine, sur une ré-exploitation des ingrédients du triangle didactique « enseignant - élève - savoir » sous la forme « figures d'action internes - figures d'action externes - objet d'enseignement et gestes didactiques ». Et si la centration sur l'un ou l'autre de ces éléments est toujours possible et légitime, c'est bien entendu leur articulation qui constitue, à nos yeux, le gage d'une véritable analyse des pratiques enseignantes.

\title{
Références bibliographiques
}

Aeby-Daghé, S. \& Dolz, J. (2008). Des gestes didactiques fondateurs aux gestes spécifiques à l'enseignementapprentissage du texte d'opinion. In D. Bucheton \& O. Dezutter (Ed.), Le développement des gestes professionnels dans l'enseignement du français (pp. 83-105). Bruxelles : De Boeck Université.

Bernié, J.-P. \& Goigoux, R. (2005). Quels concepts pour quelle analyse de l'activité des enseignants du français ? La Lettre de l'AiRDF, 36, 3-4.

Blanchet, A. \& al. (1985). L'entretien dans les sciences sociales. Paris : Dunod.

Blanchet, A. \& Gotman, A. (1992). L'enquête et ses méthodes : l'entretien. Paris : Nathan.

Borzeix, A. \& Fraenkel, B. (Eds) (2001). Langage et travail. Communication, cognition, action. Paris : CNRS.

Boutet, J. (Ed.) (1995). Paroles au travail. Paris : L’Harmattan.

Bronckart, J.-P. (1997). Activité langagière, textes et discours. Pour un interactionisme socio-discursif. Paris : Delachaux et Niestlé.

Bronckart, J.-P. (2001). S'entendre pour agir et agir pour s'entendre. In J.-M. Baudouin \& J. Friedrich (Eds), Théories de l'action et éducation (pp. 133-154). Bruxelles : De Boeck.

Bronckart, J.-P. (2008). Genres de textes, types de discours et "degrés" de langue. Hommage à François Rastier. Texto ! [En ligne], Dialogues et débats. URL : http://www.revue-texto.net/index.php?id=86.

Bronckart, J.-P. \& Groupe LAF (Eds) (2004). Agir et discours en situation de travail, Genève, Cahiers de la Section des sciences de l'Education, 103.

Bronckart, J.-P. \& Machado, A.-R. (2005). En quoi et comment les textes prescriptifs prescrivent-ils ? Analyse comparative de documents éducatifs brésiliens et genevois. In L. Filliettaz \& J.-P. Bronckart (Eds), L'analyse des actions et des discours en situation de travail. Concepts, méthodes et applications (pp. 221-240). Louvain-LaNeuve : Peeters. 
Bronckart, J.-P., Bulea, E. \& Fristalon, I. (2004). Les conditions d'émergence de l'action dans le langage. Cahiers de Linguistique Française, 26, 345-369.

Brousseau, G. (1988). Le contrat didactique : le milieu. Recherches en Didactique des Mathématiques, 3 (9), 309336.

Bucheton, D. (Ed). (2009). L'agir enseignant : des gestes professionnels ajustés. Toulouse : Octarès.

Bulea, E. (2007). Le rôle de l'activité langagière dans l'analyse des pratiques à visée formative. Thèse de doctorat. Université de Genève.

Bulea, E. (2009). Types de discours et interprétation de l'agir : le potentiel développemental des « figures d'action». Estudos Linguísticos / Linguistics Studies, 3, Ediçoes Colibri/CLUNL Lisboa, 135-152.

Bulea, E. (2010). Linguagem e efeitos desenvolvimentais da interpretação da atividade [Langage et effets développementaux de l'interprétation de l'activité.] Sao Paulo : Mercado de letras.

Bulea, E. \& Bronckart, J.-P. (2010). Les conditions d'exploitation de l'analyse des pratiques pour la formation des enseignants. Linguarum Arena. Revista do Programa Doutoral em Didáctica de Línguas da Universidade do Porto, vol. $1, n^{\circ} 1,43-60$. http://ler.letras.up.pt/site/default.aspx?qry=id04id1339id2317\&sum=sim

Bulea E. \& Bronckart J.-P. (2012). Les représentations de l'agir enseignant dans le cadre du genre « entretien». Raído, Revista Semestrial do Programa de Pós-Graduação em Letras da Universidade Federal da Grande Dourados-UFGD, v. 6, n. 11, 131-149.

Bulea, E \& Fristalon I. (2004). Agir, agentivité et temporalité dans des entretiens sur le travail infirmier. In J.-P. Bronckart et Groupe LAF (Ed.), Agir et discours en situation de travail. Genève : Cahier de la Section des Sciences de l'Education, 103.

Bulea, E. \& Jusseaume, S. (sous presses). Figures d'action et interprétation des dimensions didactiques de l'agir enseignant. LIDIL, 49.

Chiss, J.-L, David, J. \& Reuter, Y. (dir) (2005). Didactique du français. Fondements d'une discipline. Bruxelles : De Boeck.

Cicurel, F. (2011). Les interactions dans l'enseignement des langues. Agir professoral et pratiques de classe. Paris : Didier.

Canelas-Trevisi, S. (2009). La grammaire enseignée en classe. Berne : Peter Lang.

Clot, Y. (2001). Clinique du travail et action sur soi. In J.-M. Baudouin \& J. Friedrich (Eds), Théories de l'action et éducation (pp. 255-277). Bruxelles : De Boeck.

Clot, Y. \& Faïta, D. (2000). Genres et styles en analyse du travail. Concepts et méthodes, Travailler, 4, 7-42.

Clot, Y. \& Soubiran, M. (1999). «Prendre » la classe : une question de style ? Société française, 62/63, 78-88.

Daniellou, F. (1996). L'ergonomie en quête de ses principes. Toulouse : Octarès.

Dejours, C. (1999). Le facteur humain. Paris : PUF.

Dominicé, P. (1990). L'histoire de vie comme processus de formation. Paris : L'Harmattan.

Goigoux, R. (2002). Analyser l'activité d'enseignement de la lecture: une monographie. Revue Française de Pédagogie, 138, 125-134.

Grosjean, M. \& Lacoste, M. (1999). Communication et intelligence collective. Le travail à l'hôpital. Paris : PUF.

Jaubert, M. \& Rebière, M. (2006). Gestes professionnels et apprentissage de la lecture en formation des maîtres, étude de cas. In Actes du $9^{\text {ème }}$ colloque international de l'AIRDF Le français, discipline singulière, plurielle ou transversale. Québec, août 2004.

Jobert, G. (2002). La professionnalisation, entre compétences et reconnaissance sociale. In M. Altet, L. Paquay et Ph. Perrenoud (Eds), Formateurs d'enseignants. Quelle professionnalisation? (pp. 247-259). Bruxelles : De Boeck.

Jusseaume, S. (2012). L'influence des caractéristiques du public sur la représentation de l'activité de formateurs en FLE. Une approche discursive. Mémoire de maîtrise, Université de Genève.

Oddone, I., Re, A. \& Briante, G. (1981). Redécouvrir l'expérience ouvrière. Vers une autre psychologie du travail? Paris : Editions sociales.

Pineau, G. (1985). L'autoformation dans le cours de la vie : entre l'hétéro et l'écoformation, Education permanente, 78/79, 25-39.

Plane, S. \& Schneuwly, B. (2000). Regards sur les outils de l'enseignement du français : un premier repérage. Repères, 22, 3-17 [numéro coordonné par S. Plane et B. Schneuwly, «Les outils d'enseignement du français »].

Saujat, F. (2002). Ergonomie de l'activité enseignante et développement de l'expérience professionnelle: une approche clinique du travail du professeur. Thèse de doctorat. Université Aix-Marseille I.

Scheller, L. (2000). L'élaboration de l'expérience au travail. La méthode des instructions au sosie dans le cadre d'une formation universitaire. Education permanente, 146, 161-174.

Schneuwly, B. \& Dolz, J. (Dir) (2009). Des objets enseignés en classe de français. Rennes : Presses Universitaires de Rennes.

Schneuwly, B. (2009). Le travail enseignant. In : B. Schneuwly \& J. Dolz, Des objets enseignés en classe de français (pp. 29-43). Rennes : Presses Universitaires de Rennes. 
Schön, D. A. (1983/1994). Le praticien réflexif. A la recherche du savoir caché dans l'agir professionnel. Québec : Editions Logiques.

Sensevy, G. (1998). Lecture, écriture et gestes professionnels. Repères, 18, 123-136.

Sensevy, G. (2005). Sur la notion de geste professionnel. La Lettre de l'AiRDF, 36, 4-6.

Sensevy, G., Mercier, A. \& Schubauer-Leoni, M.-L. (2000). Vers un modèle de l'action didactique du professeur à propos de la course à 20. Recherches en Didactique des Mathématiques, 20(3), 263-304.

Simard, Cl., Dufays, J.-L, Dolz, J. \& Garcia-Debanc, C. (2010). Didactique du français langue première. Bruxelles : De Boeck.

Vermersch, P. (1994). L'entretien d'explicitation en formation initiale et en formation continue. Paris : ESF.

\footnotetext{
${ }^{1}$ Nous tenons à remercier ici les relecteurs/relectrices de cet article, dont les commentaires nous ont beaucoup aidée à améliorer la version initiale de ce texte.

${ }^{2}$ Pour le choix, l'identification et la définition de ces dimensions didactiques, nous prenons appui sur le second regroupement de recherches, ainsi que sur un ensemble de conceptualisations proposées dans des ouvrages qui fournissent des éléments généraux de base pour la compréhension du domaine de l'enseignement du français (voir, par exemple, Chiss, David \& Reuter, 2005 ; Simard, Dufays, Dolz \& Garcia-Debanc, 2010). L'appui sur de ce type d'ouvrages nous parait méthodologiquement intéressante, dans la mesure où, de par leur statut, ils sont susceptibles de rassembler des éléments (notions, concepts, principes, etc.) dont le statut didactique n'est pas/plus remis en question, bien qu'il n'y ait pas forcément consensus en ce qui concerne leurs définitions.

${ }^{3}$ Dans les extraits que nous allons proposer par la suite, ces formateurs seront identifiés par des initiales fictives : HT, MB, RV, GS, VS, etc. L’institution d'appartenance sera mentionnée en utilisant les abréviations I1, I2 et I3.

${ }^{4}$ Tous les exemples qui suivent sont extraits de nos données.

5 "Censé » car il arrive bien entendu que l'interviewé ne puisse pas toujours tenir cette posture, et revienne à des formulations en «je ». Nous y reviendrons dans nos analyses.

${ }^{6}$ Dans les instructions au sosie, le «je » est remplacé par «tu», ce changement étant requis par la consigne et la technique mêmes. Il s'agit dans ce cas d'une actorialité déléguée, mais qui reste de l'ordre de l'actorialité, et qui contraste fortement avec les «tu » impersonnels, par exemple, qui sont proches de « on » et que l'on peut rencontrer dans d'autres figures d'action.

${ }^{7}$ Dans les exemples de figures d'action, nous avons mis en italiques les unités ou structures qui sont propres à la figure d'action concernée, ou qui la caractérisent. Les conventions de transcription sont les suivantes : / //// : pauses de durée variable; no:::n: allongements vocaliques; deman-: unité inachevée; xxx: segments inaudibles; soulignements : chevauchements; gras : prononciation appuyée; $\uparrow \downarrow$ : intonations montante et descendante ; [entre crochets]: brèves interventions d'un interlocuteur dans le tour de parole de l'autre; (entre parenthèses): commentaires du transcripteur. Le chercheur est noté INT.

$8 \mathrm{Au}$ plan théorique, la distinction entre entre figures d'action internes et externes ré-exploite et prolonge la distinction entre action interne et action externe proposée par Bronckart (2001): l'action interne concerne les représentations et les évaluations qu'une personne se construit de son propre agir, de sa propre responsabilité dans l'activité collective, comportant ainsi des éléments (intentions, raisons, capacités, déterminations, résultats, etc.) autoreprésentés; tandis que l'action externe a trait aux représentations et évaluations de la responsabilité d'une personne telles qu'elles sont proposées par d'autres protagonistes de l'activité collective, venant donc "de l'extérieur », et comportant ainsi des éléments hétéro-représentés.

${ }^{9}$ Dans ces exemples, nous avons laissé les figures internes en caractère romain, et nous avons mis en italiques les figures externes.

${ }^{10}$ Les classifications, les extensions et les dénominations de ces gestes sont différentes selon les auteurs. Ce n'est pas le lieu ici de discuter ces différences; nous nous en inspirons d'ailleurs assez librement, selon ce qui nous semble pertinent pour notre propos. Ainsi, nous laissons par exemple de côté le geste de régulation, très fortement et régulièrement sollicité dans les contextes scolaires, mais bien moins concernés par les enseignements que nous examinons ici.
} 